\title{
Binary Effects on Supernovae
}

\author{
Philipp Podsiadlowski \\ Dept. of Astrophysics, University of Oxford, \\ Denys Wilkinson Building, Keble Road, Oxford, OX1 3RH, United Kingdom \\ email: podsi@astro.ox.ac.uk
}

\begin{abstract}
Here we review how binary interactions affect the final pre-supernova structure of massive stars and the resulting supernova explosions. (1) Binary-induced mass loss and mass accretion determine the final envelope structure, the mass, radius and chemical composition, which are mainly responsible for the supernova appearance and supernova (sub-)type. (2) Mass loss can also drastically change the core evolution and hence the final fate of a star; specifically, around $10 M_{\odot}$, it determines whether a star explodes in a supernova or forms a white dwarf, while for larger masses it can dramatically increase the minimum main-sequence mass above which a star is expected to collapse to a black hole. (3) Mass loss before the supernova directly affects the circumstellar medium (CSM) which can affect the supernova spectrum (e.g. account for the IIn phenomenon), produce powerful radio emission and, in extreme cases, lead to a strong interaction with the supernova ejecta and thus strongly modify the lightcurve shape; it may even be responsible for some of the superluminous supernovae that have recently been discovered.
\end{abstract}

Keywords. binaries (including multiple): close, stars: mass loss, supernovae: SN 1987A, supernova remnants

\section{Introduction}

It is well established that most stars are members of binary systems. Indeed, in a large fraction of systems, the two stars are close enough to interact directly by Roche-lobe overflow (in $30-50 \%$ of systems, depending on the mass of the system). For massive stars, the fraction of interacting binaries is even higher: recently Sana et al. (2012) showed that $\sim 75 \%$ of $\mathrm{O}$ stars are members of interacting binaries. As stars generally expand most dramatically after hydrogen core burning, while they spend most of their lifetime on the main sequence, observed systems are more likely to have not yet interacted. On the other hand, supernovae probe the final structure of massive stars; as most of these will have experienced at least one binary interaction at this point, the majority of supernovae will also have been affected by binary interactions. Indeed, the results of Sana et al. (2012) show that thinking of supernova progenitors as single stars that have evolved in isolation is not even the correct zeroth-order approximation.

In this review, we discuss the various ways by which binary interactions affect supernovae. In $\S 2$ we summarize how they affect the final envelope structure and resulting supernova appearance, and illustrate this with the important case of SN 1987A in $\S 3$. In $\S 4$ we discuss how they can alter the final fate of a star and in $\S 5$ how they shape the circumstellar environment and the observational signatures this produces.

\section{Binary Interactions and Supernova Lightcurves}

When a star fills its Roche lobe, there are two main types of main transfer: stable and unstable mass transfer. 


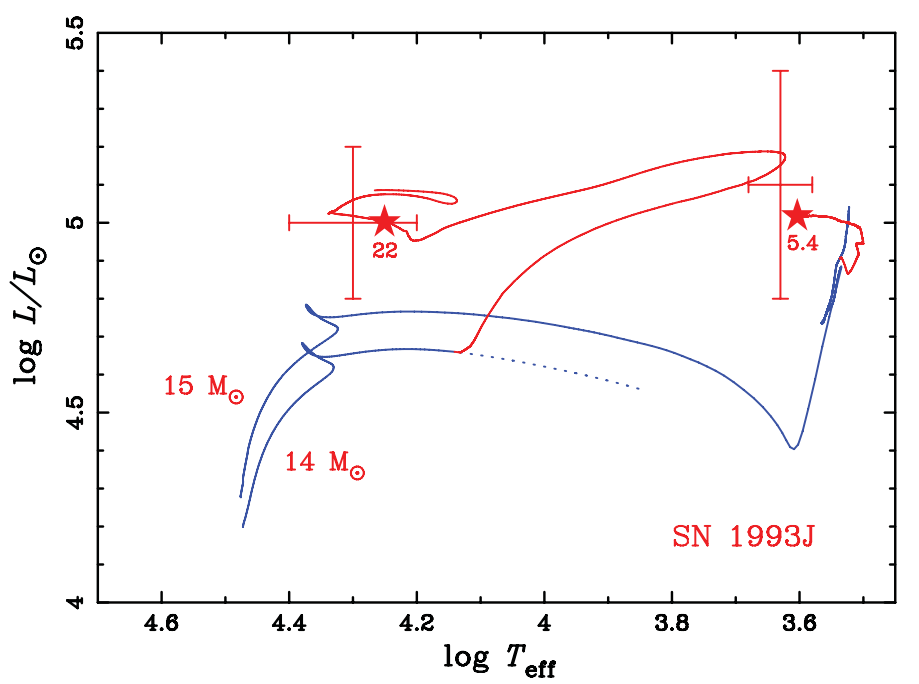

Figure 1. Hertzsprung-Russell diagram showing the evolution of the two components of a massive binary that experiences very late (so-called Case C) mass transfer. At the time of the explosion, the primary is a $5.4 M_{\odot}$ star with a small $0.3 M_{\odot}$ H-rich envelope. Because of the accreted mass the secondary never becomes a red supergiant and explodes as a blue supergiant similar to SN 1987A. The locations of the two components in the observed progenitor system of the IIb supernova SN 1993J are indicated by large error bars. (From Maund et al. 2004.)

\section{Stable Mass Transfer}

In the case of stable mass transfer, mass is transferred from one star, the donor, to the companion star, the accretor. While there may be significant mass loss from the system (in particular, at the highest mass-transfer rates), mass transfer can be largely treated as conservative, i.e. to lowest order both mass and angular momentum of the binary are conserved, and both stars are strongly affected by the mass-transfer phase. This also implies that, at the end of the mass-transfer phase, the system tends to be wider than the initial system. The mass-transfer phase generally ends when the donor star has lost most of its envelope: in the case of a hydrogen-rich donor, most of its hydrogen envelope, producing a helium (or Wolf-Rayet) star, in the case of a donor star with a helium envelope (so-called case BB mass transfer), a star without a hydrogen or helium envelope. It should be noted that generally, at the end of the mass-transfer phase, a hydrogen-rich donor star becomes detached from its Roche lobe when it still has some hydrogen left in its envelope (typically a few $0.1 M_{\odot}$ ). As this may show up in the supernova spectrum, this can lead to hybrid supernova types, such as SNe IIb (of which SN 1993J was the proto-type), which first looks like a hydrogen-rich Type II supernova, but later morphs into a SN Ib.

The further evolution of the accretor depends on the evolutionary stage at the time of mass transfer. If it was still on the main sequence, it will generally be rejuvenated, which means that it will subsequently behave like a more massive star but with its clock reset. On the other hand, if it has already left the main sequence, its core structure is now fixed, but because of the increase in the mass of the envelope, its further evolution may be drastically altered; specifically, it may never become a red supergiant, but spend most of its remaining evolution as a blue supergiant where it will explode, producing a supernova similar to SN 1987A (Podsiadlowski \& Joss 1989).

Figure 1, which represents a detailed binary evolution model for the system that produced SN 1993J (from Maund et al. 2004), illustrates the effects on both the donor and 
the accretor. At the time of the explosion, the initial primary still has a small hydrogenrich envelope, leading to a SN IIb, while the secondary, having accreted after having left the main sequence, ends its evolution as a blue supergiant similar to the case of SN 1987A.

\section{Unstable mass transfer}

Unstable mass transfer occurs when the companion cannot accrete all of the accreted mass and starts to fill and overfill its Roche lobe: essentially the envelope of the mass donor (often a giant) expands to completely engulf the companion, producing a commonenvelope system, consisting of the core of the donor and the companion star, embedded in the envelope of the donor. Because this immersed binary experiences friction with the envelope, the binary orbit shrinks, releasing orbital energy that is directly deposited in the envelope. While the details of common-envelope evolution are still poorly understood, it is clear that, in general, there are two possible outcomes:

(1) If the orbital energy is sufficient to unbind the common envelope, the latter can be ejected, stopping the spiral-in process and leaving a much closer binary, consisting of the core of the donor (again a helium star if the donor was initially hydrogen-rich) and the largely unaffected companion star.

(2) If the orbital energy is not sufficient to eject the envelope, the spiral-in process continues till the two stars have merged completely. In this case, the end product is a single star with possibly some unusual properties. It will, at least initially, be rapidly rotating (possibly having the appearance of a $\mathrm{B}[\mathrm{e}]$ supergiant), and because of the mixing induced by the merger process may have an unusual chemical profile. In addition, because of the added mass in the envelope (coming from the destroyed companion star), it may end its evolution as a blue supergiant similar to the case of a star that accreted mass (see Podsiadlowski, Joss \& Rappaport 1990 and $\S 3$ ). Also, as this may produce a rapidly rotating core at the time of the explosion, this provides a potential channel for the progenitors of gamma-ray bursts within the framework of the collapsar model (for a review see Fryer et al. 2007).

\section{Supernova types}

The envelope structure of an exploding star, its mass, radius and composition, is the main factor that determines the appearance of a supernova and hence determines its type and sub-type. For example, a progenitor with a smaller radius (such as the progenitor of SN 1987A; see $\S 3$ ) will be fainter initially because a larger fraction of the explosion energy is used up in unbinding the more tightly bound envelope. The mass of the envelope critically determines the photon diffusion time in the ejecta, which in turn determines the lightcurve width (and the length of the plateau phase in a SN II-P).

In other words, the sequence of supernova (sub-)types

$$
\mathrm{SN} \mathrm{II-P} \rightarrow \mathrm{SN} \mathrm{II-L} \rightarrow \mathrm{SN} \mathrm{IIb} \rightarrow \mathrm{SN} \mathrm{Ib} \rightarrow \mathrm{SN} \mathrm{Ic}
$$

just describes a sequence of increased mass loss, first of the hydrogen-rich envelope, then the helium envelope. As the main effect of binary interactions is to change the envelope structure (see, e.g., Podsiadlowski, Joss \& Hsu 1992), one should expect that most of the diversity along this sequence is a direct consequence of binary interactions, rather than just variations in wind mass loss in single stars. 


\section{Supernova 1987A (SN 1987A)}

SN 1987A is a particularly interesting example. While the detected neutrino signal from the collapse confirmed the basic theory of core collapse, it was highly anomalous in many respects: the star that exploded was a blue supergiant instead of a red supergiant, and the progenitor was surrounded by a complex triple-ring nebula (Wampler et al. 1990), consisting of material that was ejected by the progenitor system $\sim 20000 \mathrm{yr}$ before the explosion. The best explanation to-date is that the system initially consisted of a massive binary system that merged $20000 \mathrm{yr}$ before the explosion when part of the progenitor's envelope was ejected and subsequently swept up by the energetic blue-supergiant wind, once the merged object had become a blue supergiant (for more details see Podsiadlowski, Morris \& Ivanova 2007; Morris \& Podsiadlowski 2007; and Fitzpatrick \& Podsiadlowski [these proceedings]).

However, detailed light-echo studies by Sugerman et al. (2005) have shown that the circumstellar environment is even more complex, providing an imprint of the whole massloss history of the progenitor system. These include: (1) the fast wind in the mainsequence phase, producing a CSM bubble; (2) the slow red-supergiant wind, lasting $10^{4}-10^{5} \mathrm{yr} ;(3)$ an (early) stable mass-transfer phase before the actual merger $\left(\sim 10^{3}-\right.$ $10^{4} \mathrm{yr}$ ), in which one expects both a gravitationally flattended red-supergiant wind and a bipolar outflow from the accreting component; (4) the mass loss associated with the merger and finally (5) the energetic blue-supergiant wind.

Fitzpatrick (2012) has shown that the inclusion of all of these, in particular the redsupergiant wind and the early mass-transfer phase, can indeed explain many of the observed structures and light echoes (some of these results are presented in Fitzpatrick \& Podsiadlowski [these proceedings]).

\section{The Final Fates of Single and Binary Stars}

Binary evolution does not only affect the final envelope structure, but can also drastically change the final fate of a star. While it is clear that a star that loses mass early on the main sequence will effectively behave like a less massive star (even a star as massive as $17 M_{\odot}$ may end its life as a white dwarf; e.g. Podsiadlowski, Rappaport \& Han 2003), the effects can be even more dramatic as illustrated in Figures 2 and 3.

Figure 2 shows how the evolution of a star around $10 M_{\odot}$ is affected by the presence (in single stars) or absence of the second dredge-up phase (in the case of stars in binaries that have lost their hydrogen-rich envelopes before the AGB phase). Because the second dredge-up drastically decreases the core mass in single stars, they are more likely to end their evolution as $\mathrm{ONeMg}$ white dwarfs, rather than experience core collapse, the more likely fate for the binary case. As the supernovae produced from these objects are likely to be relatively faint electron-capture supernovae, which are not expected to impart large natal kicks to the newborn neutron stars, this may explain why low-kick neutron stars appear to prefer relatively close binary systems (see Podsiadlowski, Langer, et al. 2004 for detailed discussions).

Figure 3 illustrates how the loss of the hydrogen-rich envelope before or early during helium core burning (so-called Case B mass transfer) dramatically changes the minimum initial mass where a massive star is expected to become a black hole. As shown by Brown et al. (2001), because of the lack of a H-burning shell, the convective core does not grow during helium core burning, and stars end up with much smaller CO and ultimately iron cores. Indeed, because of this, such H-deficient stars formed in Case B binaries are expected to end their evolution as neutron stars rather than as black holes, even for 


\section{Second dredge-up in AGB stars (around 10 Msun)}

with $\mathrm{H}$ envelope

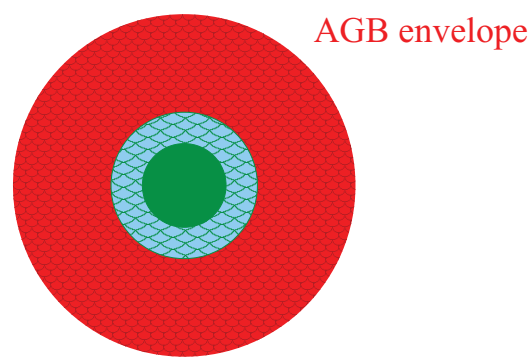

dredge-up of the He core

$\rightarrow->$ lower $\mathrm{CO}$ core masses

$\longrightarrow$ ONeMg WD

(Podsiadlowski et al. 2004) without $\mathrm{H}$ envelope

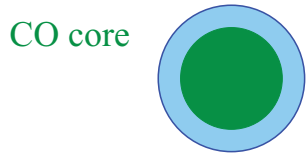

without dredge-up

$->$ larger $\mathrm{CO}$ core mass

$->$ electron-capture supernova

in $\mathrm{ONeMg}$ core

o lower explosion energy

o lower supernova kicks

o NS mass: 1.25 Msun

Figure 2. Schematic comparison of the late evolution of stars with an initial mass around $10 M_{\odot}$ with and without a H-rich envelope. The former experience a so-called second dredge-up phase at the beginning of the AGB phase reducing the size of the helium core; these stars are therefore more likely to end up as $\mathrm{ONeMg}$ white dwarfs. On the other hand, stars without the second dredge-up may eventually collapse and experience an electron-capture supernova.

initial masses as high as $\sim 60 M_{\odot}$, much larger than the likely estimated initial mass for single stars $\left(\sim 20-25 M_{\odot}\right)$.

\section{Supernova Environments and Signatures}

The complex mass loss from binary systems produces important signatures for binary interactions. The spectacular structures of many planetary nebulae, generally now believed to be largely shaped by various binary interactions, are a testament to this, as is the nebula around SN 1987A, already discussed in $\S 3$.

Figure 4 shows a simulation of how the presence of a binary companion affects the structure of a red giant in a binary (from Mohamed, Booth \& Podsiadlowski 2013). The wind is strongly focused towards the orbital plane and has the shape of a double spiral, which itself is very clumpy. These clumps can produce absorption features in highresolution supernova spectra, which will vary depending on the line of sight. Indeed, the variable $\mathrm{Na}$ lines observed in a significant fraction of Type Ia supernovae may provide a direct signature of these wind structures, which lends direct support to the idea that these systems contain red-giant donors in a single-degenerate progenitor scenario (Patat et al. 2007). Direct evidence for such spiral wind structures may also have been seen in the periodic radio lightcurves in some radio supernovae, such as SN 1979C (Weiler et al. 1991).

\section{$L B V$ Supernovae}

There has been mounting evidence in recent years (e.g. Kotak \& Vink 2006; Gal-Yam et al. 2007) that some supernovae occur in an LBV (luminous-blue-variable) phase. From a stellar evolution point of few this is very surprising as the LBV phase is believed to occur 
He-core-burning stars $(\mathrm{M}>20-25$ Msun $)$

with $\mathrm{H}$ envelope

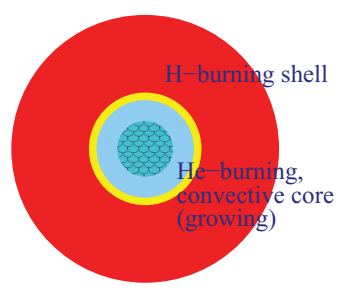

$-\rightarrow$ larger $\mathrm{CO}$ cores with lower $\mathrm{C} / \mathrm{O}$ ratio $-\rightarrow$ no convective $\mathrm{C}$ burning higher entropy (more massive) iron cores $-\rightarrow$ BLACK HOLE without $\mathrm{H}$ envelope

(up to 60/70 Msun?)

no $\mathrm{H}$-burning shell

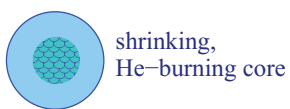

$-\rightarrow$ smaller $\mathrm{CO}$ cores with higher $\mathrm{C} / \mathrm{O}$ ratio $\rightarrow$ convective $\mathrm{C}$ burning lower entropy (mass) iron cores

\section{$-\rightarrow$ NEUTRON STAR}

(Brown, Lee, Heger)

Figure 3. Schematic comparison of the helium core-burning phase of stars with (left) and without a hydrogen envelope (right) for massive stars $\left(M>20 M_{\odot}\right)$. Because of the lack of a H-burning shell, stars without a hydrogen envelope produce smaller He-exhausted cores with a larger $\mathrm{C} / \mathrm{O}$ fraction. This makes them more likely to ultimately collapse to a neutron star instead of a black hole.
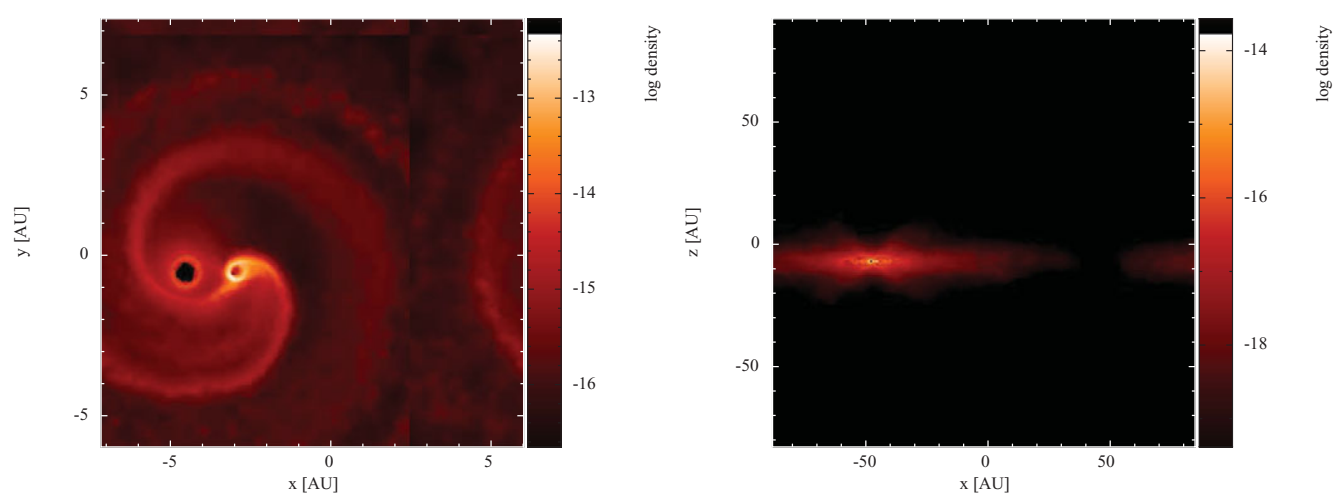

Figure 4. SPH simulations illustrating the mass loss from a symbiotic binary with a red-giant donor (simulating a system like RS Oph). The wind from the donor is assumed to be spherically symmetric, but is gravitationally focused by the companion and shaped by the orbital motion. The panels show the density structure in the orbital plane (left) and in a meridional projection (right). (From Mohamed, Booth \& Podsiadlowski 2013.)

soon (or even during) the hydrogen-core-burning phase, where massive stars lose their hydrogen-rich envelopes and become Wolf-Rayet stars, where they spend the last $>10^{5} \mathrm{yr}$ of their evolution. Indeed, if true the theory of single, massive stars would seriously need to be re-written.

Fortunately, the situation is quite different in a binary where both components merge soon after the primary's main-sequence phase: the resulting merged object will spend most of its helium-core-burning phase as a blue supergiant and only enter the LBV phase after helium core burning and may still explode in this phase (Justham, Podsiadlowski \& Vink 2013; see Fig. 5). In fact, if both stars are of similar mass and have already developed a helium-rich core, the cores may also merge, producing a much more massive 

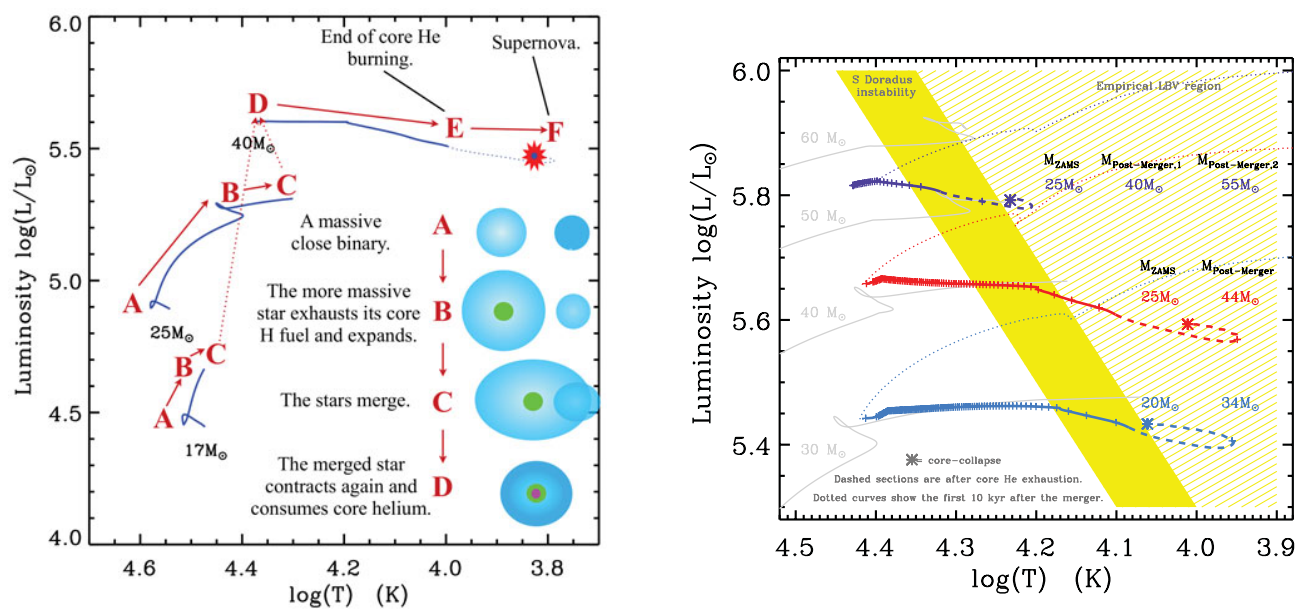

Figure 5. LBV supernovae from binary mergers. Left: schematic H-R diagram illustrating the evolution of a massive Case B merger. Right: Selected binary models, illustrating the evolution of massive mergers to the LBV phase. The most luminous case simulates a triple merger. (From Justham et al. 2013.)

core than a single, massive star could at the same metallicity. Such objects are potential candidates for pair-instability supernovae even at solar metallicity.

\section{Interaction supernovae and superluminous supernovae}

The effects of a dense circumstellar medium can be even more dramatic if the mass in the immediate neighborhood of an exploding star is a significant fraction of the ejecta mass. In this case, the interaction of the ejecta with the CSM will slow down the ejecta, efficiently converting its kinetic energy into thermal energy and ultimately radiation. If a CSM shell is at the right distance, this can power the lightcurve and may even be responsible for some of the rare, overluminous supernovae that have been discovered in recent years (Quimby et al. 2011). In this case, these supernovae would be overluminous not because they are particularly energetic, but because they are efficient in radiating their kinetic energy (Smith \& McCray 2007). One question that still remains to be answered is why does a small subset of progenitors eject a large amount of their envelopes in the last few years/decades before the explosion. One possibility is that these could be ejected common envelopes in binary systems (Chevalier 2012): depending on the precise timing of the ejection this could account for a variety of both IIn and overluminous supernovae.

\section{References}

Brown, G. E., et al. 2001, NewA, 6, 457

Chevalier, R., 2012, ApJ, 752, 2

Fitzpatrick, B., 2012, D. Phil. Thesis (Oxford University)

Fryer, C. L., et al. 2007, PASP, 119, 1211

Gal-Yam, A., et al. 2007, ApJ 656, 372

Justham, S., Posiadlowski, Ph., \& Vink, J.-S., 2013, submitted

Kotak, R. \& Vink, J. S., 2006 A $\& A$ 460, L5

Maund, J. R., Smartt, S. J., Kudritzki, R. P., Podsiadlowski, Ph., \& Gilmore, G. F., 2004, Nature, 427, 129

Mohamed, S., Booth, R., \& Podsiadlowski, Ph. 2013, in preparation

Morris, T., \& Podsiadlowski, Ph. 2007, Science, 315, 1103 
Patat, F., et al., 2007, Science, 317, 924

Podsiadlowski, Ph., \& Joss, P. C., 1989, Nature, 338, 401

Podsiadlowski, Ph., Joss, P. C., \& Hsu, J. J. L., 1992, ApJ, 391, 246

Podsiadlowski, Ph., Joss, P. C., \& Rappaport, S., 1990, A\& A, 227, L9

Podsiadlowski, Ph. \& Langer, N., et al. 2004, ApJ, 612, 1044

Podsiadlowski, Ph., Morris, T. S., \& Ivanova, N., 2007, in SN 1987A: 20 Years After, AIP Conf. Proc., Vol. 937, p. 125

Podsiadlowski, Ph., Rappaport, S., \& Han, Z., 2003, MNRAS, 341, 385

Quimby, R. M., et al. 2011, Nature, 474, 487

Sana, H., et al. 2012, Science, 337, 444

Smith, N., \& McCray, R. 2007, ApJL, 671, L17

Sugerman, B. E. K., et al. 2005, ApJS, 159, 60

Wampler, E. J., et al. 1990, ApJL, 362, 13

Weiler, K. W., et al. 1991, ApJ, 380, 161

\section{Discussion}

RAY: 1. Why do the LBV-phase reached only for stellar binaries with a lower mass threshold; what role do the initial separations play in reaching this end stages? 2. Do the LBV states reaching BSG, YSG or RSG's have differences in the stellar wind speeds?

Podsiadlowski: 1 . The lower-mass constraint arises from the assumption that you want the post-merger mass to be large enough that the system will experience LBV outbursts later. The initial separation is constrained by the requirement that the system experiences early case of mass transfer. 2. Yes, presumably the wind velocities for the different progenitors will have different wind velocities, but this will depend on the detailed physics of the LBV phenomenon that is not really understood and we did not model.

Chiotellis: Type Ia SNR, look rather spherical symmetric. The CSM of the symbiotic $\mathrm{R} \mathrm{Ne}$ are rather asymetric. Do you expect the interaction of the SN ejecta with such an AM will lead to a spherical symmetric SNR?

PodsiAdlOWsKI: Indeed the CSM is asymetric but the mass contained in the medium is much smaller compared to ejecta mass. So it is not expected substantial effects on the symmetry of the resulting SNR. 